\title{
Amplifying continuous wave phase conjugate mirror with strontium barium niobate
}

\author{
Baruch Fischer, Mark Cronin-Golomb, Jeffrey O. White, and Amnon Yariv \\ California Institute of Technology, Pasadena, California 91125
}

Ratnakar Neurgaonkar

Rockwell International, Thousand Oaks, California 91360

(Received 25 January 1982; accepted for publication 26 February 1982)

\begin{abstract}
We report the use of $\mathrm{Sr}_{1-x} \mathrm{Ba}_{x} \mathrm{Nb}_{2} \mathrm{O}_{6}$ as an efficient holographic four-wave mixing medium. We have used this material in a continuous wave phase conjugate mirror with reflectivity exceeding unity, and in a phase conjugate resonator.
\end{abstract}

PACS numbers: 42.65. - k, 42.70.Fh, 42.60.Da, 42.40. - i

An increasing amount of the research in phase conjugate optics ${ }^{1}$ has been directed recently to the study of photorefractive crystals such as $\mathrm{Bi}_{12} \mathrm{SiO}_{20}$ (Ref. 2) and more recent$\mathrm{ly}, \mathrm{BaTiO}_{3} \cdot{ }^{3,4}$ This is due mostly to the fact that these materials hold, at the moment, the best prospect for real-time image processing, such as convolution and correlation, ${ }^{5}$ edge enhancement, ${ }^{6}$ and image amplification ${ }^{7}$ at moderate $\mathrm{cw}$ power levels.

Using $\mathrm{BaTiO}_{3}$ with its large mixing efficiency led to the demonstration of phase conjugate reflectivity exceeding unity and continuous wave phase conjugate resonators ${ }^{3}$ as well as to new optical oscillator configurations including unidirectional ring oscillators. ${ }^{4}$ Until recently, the only photorefractive crystal demonstrated to be efficient enough for use in a high reflectivity phase conjugate mirror was $\mathrm{BaTiO}_{3}$, with samples produced by a single source in the world. ${ }^{8}$

We report here the first use of $\mathrm{Sr}_{1-x} \mathrm{Ba}_{x} \mathrm{Nb}_{2} \mathrm{O}_{6}$ (SBN) as an efficient photorefractive four-wave mixing medium. We have observed phase conjugate reflectivities exceeding unity in a crystal with $x=0.4$ and constructed a phase conjugate resonator in which an oscillation beam was seen to build up between the phase conjugate mirror of Fig. 1 and an ordinary plane mirror facing it. The crystal was pumped by an argon laser delivering milliwatts at $476.5 \mathrm{~nm}$. It measured $7 \times 5 \times 5 \mathrm{~mm}$, and was poled into a single domain so that the $c$ axis was parallel to the 7 -mm side.

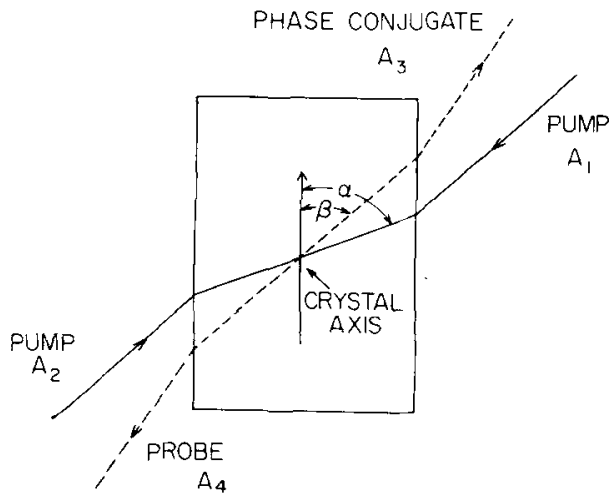

FIG. 1. Four-wave mixing arrangement appropriate to a phase conjugate mirror, showing the pump beams (solid) and probe and phase conjugate beams (dashed), as well as the relative orientation of the $c$ axis of the crystal. In the phase conjugate resonator a plane mirror is provided to retroreflect the phase conjugate beam.
The early history of SBN, like that of $\mathrm{BaTiO}_{3}$, includes its use as a holographic storage medium. ${ }^{9}$ In attempts to optimize the holographic sensitivity of photorefractive crystals in general, and SBN in particular, studies have been made of the effects of applied electric fields ${ }^{9}$ and crystal doping. ${ }^{10}$ An electric field $E_{0}$, parallel to the $k$ vector of the grating modifies the charge carrier transport behavior so as to enhance the recording sensitivity. Crystal doping increases the pool of charge carriers, since the wavelength of the writing beams is generally longer than that of the intrinsic band gap of the material. By choosing crystals with large electro-optic coefficients, the link between space charge and refractive index grating can be made stronger.

Now it is exactly these refractive index gratings which are responsible for four-wave mixing in photorefractive crystals, and it is with the electro-optic tensor and doping, or impurities in the crystal in mind that we selected SBN as a likely candidate for efficient four-wave mixing and phase conjugation. In a recent paper, ${ }^{11}$ we examined the steadystate phase conjugate reflectivity $R$ of a photorefractive phase conjugate mirror (Fig. 1) which, in the undepleted pumps and single grating approximations, ${ }^{11}$ is given by

$$
R=\left|\frac{\sinh (\gamma l / 2)}{\cosh \left(\frac{1}{2} \gamma l+\frac{1}{2} \ln r\right)}\right|^{2},
$$

where $l$ is the length of the crystal (Fig. 1), $r$ is the ratio of the intensities of the two pumping beams, and

$$
\gamma=i \omega n e^{i \phi} / 2 c \cos \theta
$$

where $\theta=90-\beta$ is the angle between the probe and the normal to the crystal (Fig. 1) and $\phi$ is phase shift between the grating and the light interference pattern. The parameter $n$ is proportional to the effective electro-optic coefficient $r_{\text {eff }}$. Since both $\mathrm{BaTiO}_{3}$ and $\mathrm{SBN}$ belong to the point group $4 \mathrm{~mm}$, this effective electro-optic coefficient is given by

$$
r_{\text {eff }}=r_{13} \sin [(\alpha+\beta) / 2] \text {, }
$$

for mixing beams of ordinary polarization and

$$
\begin{aligned}
r_{\text {eff }}= & \left\{n_{e}^{4} r_{33} \sin \alpha \sin \beta+2 n_{e}^{2} n_{0}^{2} r_{42} \cos ^{2}\left[\frac{1}{2}(\alpha+\beta)\right]\right. \\
& \left.+n_{0}^{4} r_{13} \cos \alpha \cos \beta\right\} \sin \left[\frac{1}{2}(\alpha+\beta)\right] / n_{e} n_{0}^{3},
\end{aligned}
$$

for mixing beams of extraordinary polarization, where $\alpha$ and $\beta$ are the angles of the pump beams and phase conjugate beams with respect to the $c$ axis of the crystal, as shown in Fig. 1. The $r_{i j}$ are the electro-optic coefficients, and $n_{0}$ and $n_{e}$ 
are the ordinary and extraordinary refractive indices, respectively.

When two beams intersect in a photorefractive crystal, they write an index grating and, in general, this grating couples power from one beam to the other. We performed such an experiment, coupling beams $A_{2}$ and $A_{3}$ (Fig. 1 when the other two beams are absent). From the theory of this effect ${ }^{12}$ we derived a coupling strength comparable with the value which would produce a phase conjugate reflectivity $R$ equal to one; that is $|\gamma l|=1.762$. This is the threshold for a phase conjugate resonator.

Now, in the case of $\mathrm{BaTiO}_{3}$, high phase conjugate reflectivities were obtained by taking advantage of the large electro-optic coefficient ${ }^{13} r_{42} \sim 820 \times 10^{-12} \mathrm{mV}^{-1}$. On inspection of expressions (3) and (4) we see that this necessitated first the use of extraordinarily polarized light and second, $(\alpha+\beta) / 2$ could not be a multiple of $\pi / 2$. That is, the grating wave vector cannot be parallel to any crystal axis. This, together with the high refractive index of $\mathrm{BaTiO}_{3}\left(n_{e}=2.42\right)$, made it necessary to orient $(100)$ cut crystals at large angles with respect to the incident beams. (100) cut crystals are used because during poling, it is necessary to apply an electric field parallel to the $c$ axis. For SBN, however, the largest electro-optic coefficient is $r_{33}$, which measured $\sim 200 \times 10^{-12} \mathrm{mV}^{-1}$ for the sample in which we observed high reflectivity. The grating wave vector could thus be parallel to the $c$ axis, so that the beams could be incident at nearnormal angles to the crystal surface. When we demonstrated the phase conjugate resonator we used $\alpha=83^{\circ} \beta=78^{\circ}$ because of restrictions imposed by optical inhomogeneities (striae) due to stress in the crystal. These inhomogeneities tended to scatter beams travelling in some directions more than others.

In this letter we have reported high phase conjugate reflectivities in $\mathrm{Sr}_{1-x} \mathrm{Ba}_{x} \mathrm{Nb}_{2} \mathrm{O}_{6}$ for $x=0.4$. However, we feel that even better results will be obtained for lower values of $x$. As the concentration of barium decreases, the paraferroelectric phase transition temperature drops to $T_{c}$ $\sim 56^{\circ}$ Cat $x=0.25$ which represents the lowest stable concentration at barium obtainable in SBN. The closer the operating temperature is to $T_{c}$ the larger the electro-optic coefficient becomes, especially for the case where the low-frequency electric field is along the $c$ axis which is the direction of spontaneous polarization. The electro-optic behavior of oxygen-octahedra ferroelectrics is described by the constants $g_{i j} \cdot{ }^{14,15}$ In terms of them, the electro-optic tensor is given by

$$
\begin{aligned}
& r_{33}=\left(2 g_{11} / \zeta^{3}\right) \epsilon_{0}\left(\epsilon_{c}-1\right) P_{s}, \\
& r_{13}=\left(2 g_{12} / \zeta^{3}\right) \epsilon_{0}\left(\epsilon_{c}-1\right) P_{s}, \\
& r_{42}=\left(g_{44} / \xi^{3}\right) \epsilon_{0}\left(\epsilon_{a}-1\right) P_{s},
\end{aligned}
$$

$\epsilon_{c}$ and $\epsilon_{a}$ are the relative dielectric constants of the crystal parallel and perpendicular to the $c$ axis, respectively. $P_{s}$ is the spontaneous polarization and the $g_{i j}$ are given by 0.17 , 0.04 , and $0.12 \mathrm{~m}^{4} / \mathrm{C}^{2}$ for $g_{11}, g_{12}$, and $g_{44}$, respectively. $\zeta$ is the packing density of the octahedra normalized to that of perovskites. For tungsten bronzes like SBN, it is 1.06. Thus, the $r_{i j}$ increases with the $\epsilon P_{\mathrm{s}}$ as the phase transition temperature is approached. ${ }^{15}$
TABLE I. Absorption of several photorefractive crystal samples at laser wavelengths.

\begin{tabular}{lccc}
\hline \hline $\mathrm{cm}^{-1}$ & $\mathrm{BaTiO}_{3}$ & $x=0.4 \mathrm{SBN}$ & $x=0.25 \mathrm{SBN}$ \\
\hline $632.8 \mathrm{~nm}$ & 0.51 & 0 & 0 \\
& \pm 0.01 & \pm 0.01 & \pm 0.01 \\
$514.5 \mathrm{~nm}$ & 1.0 & 0.16 & 0 \\
& \pm 0.05 & \pm 0.04 & \pm 0.01 \\
$476.5 \mathrm{~nm}$ & 1.13 & 0.19 & 0.04 \\
& \pm 0.02 & \pm 0.04 & \pm 0.04 \\
\hline \hline
\end{tabular}

In SBN with $x=0.25$, the electro-optic coefficient $r_{33}$ is about $1330 \times 10^{-12} \mathrm{mV}^{-1}$ at room temperature. ${ }^{16} \mathrm{We}$ tested a crystal of SBN with this barium concentration and failed to obtain high phase conjugate reflectivity, probably because the ionizable charge carrier density was too low. This hypothesis was strengthened when we looked at the absorption in the sample (Table I). A crystal grown at Rockwell, $x=0.4$, did give high phase conjugate reflectivity, in spite of its lower electro-optic coefficient. Its room-temperature half-wave voltage of $300 \mathrm{~V}$ at wavelength of $632.8 \mathrm{~nm}$ indicated an $r_{33}$ of $\sim 200 \times 10^{-12} \mathrm{mV}^{-1}$, while the theoretical expression (5) gave $r_{33} \sim 600 \times 10^{-12} \mathrm{mV}^{-1}$ based on $P_{x}=$ $0.27^{\circ} \mathrm{C} / \mathrm{m}^{2}$ (Ref. 17) and the measured value of $\epsilon_{c}=880$. It did, however, absorb more at $476.5 \mathrm{~nm}$ than the $x=0.25$ sample, as we see in the table. We observed also that neither sample absorbed sufficiently in the red to give high phase conjugate reflectivity at the helium neon laser wavelength at $632.8 \mathrm{~nm}$ where our $\mathrm{BaTiO}_{3}$ sample, whose absorption characteristics are given in the table, still excels.

In conclusion, then, we have demonstrated the photorefractive material SBN in the construction of both a phase conjugate mirror with reflectivity greater than one, and a phase conjugate resonator. We have made some preliminary comparisons of two samples of SBN with differing barium concentrations. More complete studies ${ }^{18}$ of the effects of crystal dopants, barium concentration, and operating temperature will be the subject of future publications.

The authors wish to thank Dr. J. B. Thaxter of the Sperry Research Center of the Sperry Corporation for supplying several samples of SBN. B. Fischer would like to acknowledge the support of the Weizmann Postdoctoral Fellowship, and M. Cronin-Golomb the support of the University of Sydney. This work was supported by the Air Force Office of Scientific Research and the Army Research Office, Durham, North Carolina.

\footnotetext{
${ }^{1}$ A. Yariv, IEEE J. Quantum Electron. QE-14, 650 (1978); R. Guiliano, Phys. Today (April 1981).

${ }^{2}$ J. P. Huignard, J. P. Herriau, G. Rivet, and P. Gunter, Opt. Lett. 5, 102 (1980).

${ }^{3} J$. Feinberg and R. W. Hellwarth, Opt. Lett. 5, 519 (1980).

${ }^{4}$ J. O. White, M. Cronin-Golomb, B. Fischer, and A. Yariv, Appl. Phys. Lett. 40, 450 (1982).

${ }^{5}$ J. O. White and A. Yariv, Appl. Phys. Lett. 37, 5 (1980).

${ }^{6}$ J. Feinberg, Opt. Lett. 5, 330 (1980).

${ }^{7}$ J. P. Huignard and A. Marrakchi, Opt. Lett. 6, 622 (1981).

${ }^{8}$ Sanders Associates, 95 Canal St., Nashua, NH 03960.

${ }^{9} J$. B. Thaxter and M. Kestigian, Appl. Opt. 13, 913 (1974).

${ }^{10}$ K. Megumi, H. Kozuka, M. Kobayashi, and Y. Furuhata, Appl. Phys
} 
Lett. 30, 631 (1977).

${ }^{11}$ B. Fischer, M. Cronin-Golomb, J. O. White, and A. Yariv, Opt. Lett. 6, 519 (1981).

${ }^{12}$ N. V. Kukhtarev, V. B. Markov, S. G. Odulov, M. S. Soskin, and L. Vinetskii, Ferroelectrics 22, 949 (1979).

${ }^{13}$ A. Yariv, Quantum Electronics, 2nd ed. (Wiley, New York, 1975).

${ }^{14}$ A. J. Fox, J. Appl. Phys. 44, 254 (1973).
${ }^{15}$ M. D. Domenico, Jr. and S. H. Wemple, J. Appl. Phys. 40, 720(1969); M. D. Domenico, Jr. and S. H. Wemple, J. Appl. Phys. 40, 735 (1969).

${ }^{16}$ P. V. Lenzo, E. G. Spencer, and A. A. Ballman, Appl. Phys. Lett. 11, 23, (1967).

${ }^{17}$ A. M. Glass, J. Appl. Phys. 40, 4699 (1969).

${ }^{18}$ We have recently observed phase conjugate reflectivities greater than one in a sample of SBN with $x=0.5$.

\title{
Output power and temperature dependence of the linewidth of single- frequency cw (GaAl)As diode lasers
}

\author{
D. Welford and A. Mooradian \\ Lincoln Laboratory, Massachusetts Institute of Technology, Lexington, Massachusetts 02173
}

(Received 4 January 1982; accepted for publication 2 March 1982)

\begin{abstract}
We report here the observation of a linear dependence of $\mathrm{cw}$ single-frequency $(\mathrm{GaAl}) \mathrm{As}$ diode laser linewidth as a function of reciprocal output power at 77,195 , and $273 \mathrm{~K}$. The observed data are explained in terms of spontaneous emission events and their related refractive index perturbations.
\end{abstract}

PACS numbers: 42.55.Bi, 42.55.Px

The use of semiconductor diode lasers as high-resolution spectral sources requires an understanding of the linewidth characteristics of these devices. Reported here is a study of the output power and temperature dependence of the spectral linewidth of $\mathrm{cw}$ single-frequency $(\mathrm{GaAl}) \mathrm{As}$ diode lasers.

The experiments were carried out on single-frequency channel substrate planar (CSP) Hitachi and transverse junction stripe (TJS) Mitsubishi diode lasers. The linewidths were observed to increase linearly with reciprocal output power ${ }^{1,2}$ with a slope significantly greater than the calculated Schawlow-Townes linewidth at all temperatures. A significant power-independent contribution to the linewidth was observed which is attributed to refractive index fluctuations resulting from statistical fluctuations in electron number in the small active volume of the device, and is presented elsewhere. ${ }^{3}$ The power-dependent linewidth data are reported here and the discrepancy between the experimental observations and the Van der Pol oscillator treatment of the Schawlow-Townes linewidth ${ }^{4}$ is explained using the enhancement factor of Henry. ${ }^{6}$

The experimental arrangement has been described elsewhere. ${ }^{3}$ The devices were thermally isolated in a Dewar to reduce temperature fluctuations to an insignificant level. Each data point for a particular power and temperature was the result of the integration of many Fabry-Perot interferometer scans using a computer. The output power was measured using a calibrated silicon photodiode, and a dual-grating (12 000 lines $/ \mathrm{mm}$ ) 3/4-m spectrometer was used to verify single-frequency operation of the laser diode. Precautions against optical feedback were taken to avoid affecting the output of the devices. Injection current noise in the diodes was minimized by using a shielded lead-acid battery based passive component power supply incorporating a 500-
$\mathrm{kHz}$ bandwidth filter network. Linewidth measurements were made using a scanning Fabry-Perot interferometer with a resolution of $3.5 \mathrm{MHz}$. The experimental data presented are for one particular TJS laser at 273, 195, and $77 \mathrm{~K}$, and are representative of the typical performance of 15 such diodes. The single-ended output power versus injection current characteristics for the diode lasers at each of the three temperatures was quite linear over the entire operating range.

The fundamental linewidth of a laser due to quantum phase fluctuations is given by the relation ${ }^{4}$

$$
2 \Gamma=\left(\pi h v \Gamma_{c}^{2} / P\right) n_{\mathrm{sp}},
$$

where $2 \Gamma$ is the full width at half-maximum of the emission line at frequency $v, P$ is the intracavity power emitted by stimulated emission, and $\Gamma_{c}$ is the passive resonator linewidth given by

$$
\Gamma_{c}=c(\alpha L-\ln R) / 2 \pi n L,
$$

where $n$ is an effective refractive index including dispersion, $\alpha$ is the mode loss coefficient, $L$ is the cavity length, and $R$ is the facet reflectivity. Equation (1) applies to the case where the driving noise source, spontaneous emission, is homogeneous and band limited.

Experimentally, we measure the single-ended output power $P_{0}$, which is related to the intracavity power $P$ by

$$
P_{0}=[\ln (\vee R) /(\alpha L-\ln R)] P .
$$

The spontaneous emission factor $n_{\text {sp }}$, which is the ratio of spontaneous emission rate per mode to the stimulated emission rate per laser photon, is for a semiconductor laser expressed as

$$
n_{\mathrm{sp}}=\left\{1-\exp \left[\left(h v+E_{F V}-E_{F C}\right) / k T\right]\right\}^{-1},
$$

where $E_{F C}$ and $E_{F V}$ are the conduction-band and valence- 